\title{
Patient and public involvement in urogynecology: a pause for reflection before taking a leap
}

\author{
Sharif Ismail • Diaa E. E. Rizk
}

Received: 10 November 2014 / Accepted: 2 February 2015 /Published online: 26 February 2015

(C) The International Urogynecological Association 2015

\section{Background}

Patient and public involvement has become an integral part of health services [1-4]. It has progressed from simple patient satisfaction surveys to public forums debating service developments and budget. Role players take part in undergraduate and postgraduate medical examinations [5], patient feedback is included in periodic appraisals of medical practitioners and regulatory bodies include lay members on their panels. Patient representatives comment on medical guidelines and research proposals [6].

The rationale for this is both professional and political [1, 2]. Professionally, hearing patient and public views enables understanding of their knowledge and perceptions in addition to the impact of illness on their lives. This helps aspects such as privacy, dignity, access, explanation and health promotion, which can be overlooked, to be appreciated [7]. This information is also valuable in teaching and training medical students and trainees in addition to performance appraisal of medical practitioners. Politically, the philosophy is to empower individuals and communities to shape and monitor health service delivery and ensure that resources are allocated according to

S. Ismail $(\bowtie)$

Brighton and Sussex University Hospitals NHS Trust, Brighton BN2 5BE, England, UK

e-mail: sharif212121@hotmail.com

S. Ismail

Brighton and Sussex Medical School, Brighton BN2 5BE, England, UK

D. E. E. Rizk

Department of Obstetrics and Gynecology, College of Medicine and Medical Sciences, Arabian Gulf University, Manama, Kingdom of Bahrain local needs. This trend originated in the United Kingdom and is spreading to other countries such as South Korea [8], Italy [9], Ireland [3], and Norway [10]. Urogynaecology has pioneered the use of quality of life questionnaires in patient evaluation and follow-up after treatment. Patient and public involvement is a requirement for unit accreditation by the British Society of Urogynaecology. Despite taking the lead in this direction, the urogynaecological community needs to critically reflect on this approach before moving further $[1,2$, $4,6]$.

\section{Who is the public?}

Whilst it is easy to recognise patients as those who present with urogynaecological problems, and their relatives and carers, the public is more difficult to define. The wider community includes potential service users who may not be aware of the services available and/or do not feel confident enough to seek help. It also includes possible future users, who may not have problems now, but might develop them in the future. Representativeness therefore becomes important. The most vocal and influential self-selected proactive members of the community tend to express their views, although they rarely need or use health services, as they are usually well educated, in good health, and have private health care provisions. On the other hand, disadvantaged groups that often need and utilize public health services are usually marginalized or excluded [9]. Representation has been questioned in the medical literature, but this has been misinterpreted as power manoeuvring by healthcare professionals [11]. In multi-ethnic communities, cultural background may influence health behaviour and attitudes to health care and language barriers may inhibit free communication and interaction. Therefore, it is imperative to 
proactively engage all groups [12]. This is pertinent as most public representatives in clinical trials come from North America and Europe, which limits the applicability of research findings to the global scene [13].

The relationship between heath care providers and service users is not always simple [2]. Patients, relatives, carers, and the public at large are not usually aware of the variables that influence heath care such as government regulations and budget constraints. This information might be difficult to understand or give imprecise impressions about priorities and leakage may undermine public confidence in health care or have political consequences. Dialogue requires good communication and negotiation skills and a basic knowledge of administration. Working with heterogeneous groups requires considerable coordination and team working, as each group has its own agenda and priorities $[1,2]$. Although health care professionals acquire some of these skills from individual interactions, they are not formally trained in strategic communication at a macro level. Opening the door for expression of demand may increase the already high expectations, which may not be possible to meet and can therefore lead to disappointment and loss of faith in health care organisations and providers [14]. It can also undermine the confidence of healthcare staff and their willingness to participate.

\section{Involvement in guidelines and research}

The principal goal of patient involvement in medical guidelines is to improve compliance with treatment, which is often poor [15]. This approach ensures taking the views of patients, relatives, and carers into consideration, so that recommended management lines are acceptable to patients and can be followed in real day to day life. This empowers patients and the public to play an active role in their health and disease management, improves community awareness of and access to health care services, and facilitates effective lay knowledge transfer and health education [6]. There is empirical evidence to support the assumption that active patient participation enhances the quality of guidelines. Moreover, the studies showed that patients experience several difficulties in the participation process that cannot solely be traced back to flawed practices [4].

As for research, patient involvement avoids wasted research with outcomes that are irrelevant to patients and their relatives and carers [16]. A recent systematic review of clinical trials in several specialities showed that only $16 \%(1: 6)$ reported that the public had been involved in selecting outcome measures; indicating the need for good methodological guidance to improve upon this [13]. The Core Outcome Measures for Effectiveness Trials (COMET) initiative entails the development and adoption of a core outcome set (COS) for each health area as a minimum for all trials. This will not only enable comparison, but will also ensure the relevance of the outcome measures to all stakeholders, especially patients and members of the public [13].

\section{Evidence of value}

Despite the plausible benefit of involving patients and members of the public, there is hardly any research in this respect, let alone any data to demonstrate such a benefit. There is no proof of effective outcome to justify the time and resources required for patient and public involvement [10]. There is insufficient experience and limited research in collective individual interaction and personal exchange without a theoretical understanding of the mechanisms underpinning the process [4]. The cost of organising involvement and making service changes in response to it is also unknown. The initial optimism with actively involving patients in medical guideline development has been re-considered after a systematic review of the literature $[4,6]$. The anticipated impact on compliance and on the long-term outcome of treatment was disappointing [6]. There is an urgent need for primary research in this area to assess its real value and establish the helping and hindering factors.

\section{Conclusion and the way forward}

The concept of patient and public partnership in health care is an important milestone that has been favourably received by the health profession. However, calls for more patient and public involvement are widely promoted at present, without an underlying socio-cultural learning theory to guide the development of methods and standards. Moreover, there are no valid or reliable methods of assessing the impact of patient and public involvement or evidence of significant improvement in service. The process itself requires fair representation of patient cohorts who are actual consumers of the service and coaching of health care providers to learn the necessary skills to co-work with patients and the public. The fear is that patient and public involvement in health service will become a "tick box" exercise [1] and spread rapidly to urogynaecology without adequate understanding or scientific evaluation.

\section{References}

1. Bradshaw PL (2008) Service user involvement in the NHS in England: genuine user participation or a dogma-driven folly? J Nurs Manag 16:673-681 
2. Martin GP (2008) Representativeness, legitimacy and power in public involvement in health-service management. Soc Sci Med 67: 1757-1765

3. Higgins A, Maguire G, Watts M et al (2011) Service user involvement in mental health practitioner education in Ireland. J Psychiatr Ment Health Nurs 18:519-525

4. Mockford C, Staniszewska S, Griffiths F, Herron-Marx S (2012) The impact of patient and public involvement on UK NHS health care: a systematic review. Int J Qual Health Care 24:28-38

5. Rees CE, Knight LV, Wilkinson CE (2007) User involvement is a sine qua non, almost, in medical education: learning with rather than just about health and social care service users. Adv Health Sci Educ 12:359-390

6. Van de Bovenkamp HM, Trappenburg MJ (2009) Reconsidering patient participation in guideline development. Health Care Anal $17: 198-216$

7. Chadwick A (2012) A dignified approach to improving the patient experience: promoting privacy and respect though collaborative training. Nurs Educ Pract 12:187-191

8. Kim YD, Ross L (2008) Developing service user involvement in the South Korean disability services: lessons from the experience of community care policy and practice in UK. Health Soc Care Commun 16:188-196
9. Cirillo I, Politi P, Rampi E, Secker J, Brasia F, Severino A (2009) Employment status, aspirations and perceptions of support amongst psychiatric service users in Pavia, Italy. Epidemiol Psichiatr Soc 18:40-47

10. Storm M, Hausken K, Mikkelsen A (2010) User involvement in inpatient mental health services: operationalisation, empirical testing and validation. J Clin Nurs 19:1897-1907

11. Felton A, Stickley T (2004) Pedagogy, power and service user involvement. J Psychiatr Ment Health Nurs 11:89-98

12. Cross-Sudworth F, Williams A, Herron-Marx S (2011) Maternity services in multi-cultural Britain: using Q methodology to explore the views of first- and second-generation women of Pakistani origin. Midwifery 27:458-468

13. Gargon E, Gurung B, Medley N et al (2014) Choosing important health outcomes for comparative effectiveness research: a systematic review. PLoS One 9(6):e99-e111

14. Cotterell P, Harlow G, Morris C, Beresford P, Hanley B, Sargeant A, Sitzia J, Staley K (2011) Service user involvement in cancer care: the impact on service users. Health Expect 14:159-169

15. World Health Organisation (2010) People-Centered Healthcare Initiative, National Indicators Project, World Health Organization, Western Pacific Region, Geneva

16. Chamlers I, Glasziou P (2009) Avoidable waste in the production and reporting of research evidence. Lancet 374:86-89 